\title{
PREPARAÇÃO E CARACTERIZAÇÃO DO COMPLEXO COBALOXIMA E SUA UTILIZAÇÃO NA CONSTRUÇÃO DE UM ELETRODO MODIFICADO. UM EXPERIMENTO ELETROQUÍMICO NO CURSO DE GRADUAÇÃ̃O
}

\author{
Claudemir A. Borgo, Angélica M. Lazarin, Celso U. Davanzo e Yoshitaka Gushikem* \\ Instituto de Química, Universidade Estadual de Campinas, CP 6154, 13084-971 Campinas - SP
}

Recebido em 2/9/02; aceito em 2/4/03

\begin{abstract}
PREPARATION AND CHARACTERIZATION OF THE COBALOXIME COMPLEX AND ITS USE IN FABRICATION OF A MODIFIED ELECTRODE. AN ELECTROCHEMICAL EXPERIMENT FOR UNDERGRADUATE COURSE. The present experiment describes the preparation, characterization of n-butyl(pyridil)cobaloxime complex and its electrochemical property. The infrared and uv-visible absorption spectra were used to characterize the complex obtained. The infrared spectrum of the compound showed characteristics bands that indicated the formation of the Co-C chemical bond formation. The electronic absorption spectrum in acetonitrile showed transition bands attributed to $\pi-\pi^{*}$, metal-to-ligand charge transfer, $\mathrm{d}$-d transitions and charge transfer Co-C. The electrochemical property was investigated by the pulse differential voltammetry technique. Two oxidation processes: $\mathrm{Co}(\mathrm{I}) / \mathrm{Co}$ (II) at $-423 \mathrm{mV}$ and $\mathrm{Co}(\mathrm{II}) / \mathrm{Co}(\mathrm{III})$ at $752 \mathrm{mV}$ were observed.
\end{abstract}

Keywords: n-butyl(pyridil)cobaloxime; cobalt ion; pulse differential voltammetry.

\section{INTRODUÇÃO}

Cobaloxima é a denominação comumente usada para identificar o esqueleto bis(dimetilglioximato)cobalto(III), onde 4 átomos de nitrogênio estão coordenados ao íon cobalto, formando uma estrutura quadrado planar ${ }^{1}$. Um radical orgânico R (butila) e uma base de Lewis (piridina) completam a estrutura octaédrica distorcida, resultando em um composto eletricamente neutro e estável (butil(piridil)cobaloxima), Figura 1. Cobaloximas tornaram-se compostos de grande importância, mesmo sabendo que não estão presentes na coenzima $\mathrm{B}_{12}$, mas como vantagem apresenta-se como um composto modelo da mesma. A cobaloxima é uma corrina complexada a $\mathrm{Co}^{\mathrm{I}}$ e possui em sua estrutura uma ligação cobalto-carbono (Co-C). Estes compostos foram extensivamente estudados para a elucidação do mecanismo que controla a atividade do complexo $\mathrm{B}_{12}{ }^{1-7}$. Além disso, as cobaloximas reúnem importantes propriedades que as tornam excelentes modelos para uso em laboratório de ensino, sendo possível demonstrar diversos aspectos da Química. Pode-se utilizá-las para enfatizar técnicas especiais de síntese, estudos de reatividade e ca-

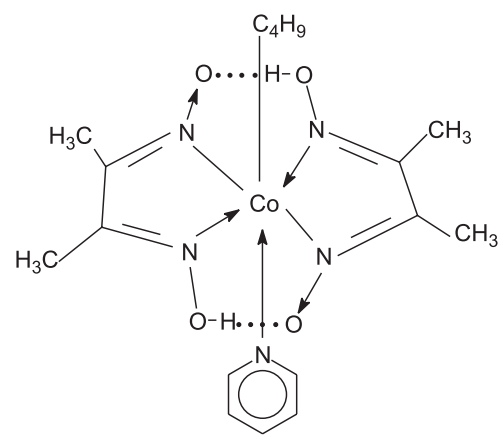

Figura 1. Estrutura da n-butil(piridil)cobaloxima

\footnotetext{
*e-mail: gushikem@iqm.unicamp.br
}

racterização utilizando-se métodos espectroscópicos e eletroquí$\operatorname{micos}^{8-9}$. Pode-se destacar como tópicos a serem analisados:

a) formação da ligação $\mathrm{Co}-\mathrm{C}$, que torna as cobaloximas exemplos de compostos organometálicos,

b) o íon cobalto apresenta-se nos estados de oxidação I a III nas diferentes etapas da síntese que podem ser executadas em laboratório sem infra-estrutura especial,

c) os reagentes são baratos e de fácil aquisição,

d) as diferentes etapas da síntese podem ser controladas e acompanhadas pelas mudanças de cor,

e) a adequada escolha do radical orgânico $\mathrm{R}$ permite estudo de reações fotoquímicas, também sem necessidade de aparato especial e,

f) há uma série de caracterizações físico-químicas que podem ser executadas dependendo da infra-estrutura disponível no laboratório.

Nesta publicação descreve-se um experimento realizado na disciplina Química Inorgânica Experimental, ministrada para estudantes do penúltimo semestre do curso de Química da UNICAMP. No experimento utilizou-se a butil(piridil)cobaloxima, composto organometálico que, apesar de ser sintetizado em condições especiais, pode ser caracterizado por técnicas tradicionais como as espectroscopias no infravermelho (iv) e no ultra-violeta-visível (uvvis). Nas cobaloximas o íon cobalto pode se encontrar em diversos estados de oxidação, esta é uma oportunidade única de observar eletroquimicamente a transição entre estes estados. A voltametria cíclica tradicional não é adequada para o estudo proposto, de modo que se empregou a voltametria de pulso diferencial. Além de ser uma técnica de fácil acesso ela poderá ser aplicada, se desejado, em conjunção com a voltametria cíclica tradicional no sistema $\left[\mathrm{Fe}(\mathrm{II})(\mathrm{CN})_{6}\right]^{4}$ $/\left[\mathrm{Fe}(\mathrm{III})(\mathrm{CN})_{6}\right]^{3-}$ para efeito de comparação $\mathrm{O}^{10-12}$. Os eletrodos geralmente empregados para os estudos eletroquímicos no estado sólido são denominados eletrodos quimicamente modificados (EQM) e, entre eles, destacam-se os eletrodos de ouro, platina, grafite pirrolítico e os eletrodos de pasta de carbono (EPC). Os eletrodos de pasta de carbono possuem vantagens adicionais em relação aos demais como, por exemplo, baixa corrente de fundo, baixo ruído, baixo custo, facilidade de renovação de superfície, além de permitirem a incorpora- 
ção de suportes e mediadores, aumentando a variedade de aplicações, contornando problemas como, a baixa reprodutibilidade e estabilidade.

Como a aplicação da eletroquímica para caracterização de compostos inorgânicos é pouco utilizada, resolveu-se relatar a experiência dos autores com este tema e, segundo nosso conhecimento, está sendo abordado pela primeira vez em curso de graduação.

\section{PARTE EXPERIMENTAL}

\section{Preparação da n-butilcobaloxima ${ }^{1}$}

Em um balão de três bocas de $500 \mathrm{~mL}$, conectou-se em uma das bocas um condensador de refluxo (não sendo necessário refrigeração), em uma boca lateral adaptou-se um "top" onde manteve-se um fluxo contínuo de gás inerte (nitrogênio ou argônio) e na terceira boca, uma tampa esmerilhada. Ao balão adicionou-se $100 \mathrm{~mL}$ de metanol, mantendo-se a deaeração e agitação por aproximadamente $10 \mathrm{~min}$. Em seguida, adicionou-se $0,80 \mathrm{~mL}$ de piridina $(11 \mathrm{mmol}) \mathrm{e}$ $2,32 \mathrm{~g}$ de dimetilglioxima (20 mmol). Após dissolução de todo o sólido adicionou-se $2,37 \mathrm{~g}$ de $\mathrm{CoCl}_{2} \cdot 6 \mathrm{H}_{2} \mathrm{O}(10 \mathrm{mmol})$. Verificou-se o aparecimento de uma coloração alaranjada. Depois de 5 min, adicionou-se vagarosamente uma solução de $\mathrm{NaOH}$, obtida através da dissolução de 1,6 g de $\mathrm{NaOH}$ (40 mmol) em $5 \mathrm{~mL}$ de água destilada. Observou-se que a suspensão tornou-se azul escuro. Entretanto, se após adição da solução de $\mathrm{NaOH}$ a suspensão tornar-se alaranjada, a reação deve ser suspensa e reiniciada, pois indica a formação do produto indesejado. Isto ocorre devido à presença de oxigênio atmosférico ou a impurezas do gás inerte (nitrogênio ou argônio utilizado).

À suspensão azul escuro adicionou-se 0,60 mL de 1-bromobutano ( $5 \mathrm{mmol}$ ) dissolvido em $5 \mathrm{~mL}$ de metanol. Após alguns segundos verificou-se que a solução tornou-se alaranjada escura. Transferiuse o conteúdo do balão para um béquer de $1000 \mathrm{~mL}$ contendo uma mistura de aproximadamente $400 \mathrm{~mL}$ de gelo e água. Agitou-se vigorosamente a solução e deixou-se em repouso por aproximadamente $30 \mathrm{~min}$ até a formação de precipitado. Filtrou-se o precipitado obtido em um funil de büchner e lavou-se o sólido com excesso de água destilada até o filtrado recolhido adquirir coloração amarelo pálido. O sólido obtido foi seco em linha de vácuo $\left(10^{-3} \mathrm{mPa}\right)$ à temperatura ambiente por aproximadamente $1 \mathrm{~h}$.

Observação: O sólido não deve ser lavado com etanol, pois este é solúvel no mesmo.

\section{Espectroscopia na região do infravermelho}

O espectro infravermelho foi registrado na região de 4000 a $400 \mathrm{~cm}^{-1}$ utilizando-se um espectrofotômetro FT-IR Bolmen Hartmann \& Braum, modelo MB 100 e o material prensado em pastilha de $\mathrm{KBr}$ a $1 \%$.

\section{Espectros eletrônicos}

Os espectros eletrônicos da n-butil(piridil)cobaloxima foram obtidos dissolvendo-a em acetonitrila, $\left(\mathrm{CH}_{3} \mathrm{CN}\right)$. Utilizou-se uma cela de quartzo de $1 \mathrm{~cm}$ de caminho ótico e um espectrofotômetro Beckman DU 640.

\section{Voltametria de pulso diferencial}

\section{Construção do eletrodo de trabalho}

Para caracterização do comportamento voltamétrico da nbutil(piridil)cobaloxima utilizou-se um eletrodo de pasta de carbo- no. Geralmente, os eletrodos de pasta de carbono consistem em uma mistura de grafite, o material de interesse e um óleo mineral (Nujol). As quantidades de material para a construção do eletrodo utilizado foram: $0,010 \mathrm{~g}$ de $\mathrm{n}$-butil(piridil)cobaloxima, a mesma proporção de grafite (Aldrich, granulometria > 300 mesh), previamente seco em estufa a $420 \mathrm{~K}$ por $2 \mathrm{~h}$ e uma gota de nujol. A pasta obtida foi inserida em um tubo de vidro de aproximadamente $5 \mathrm{~mm}$ de diâmetro interno, o qual contém uma placa de platina com aproximadamente $2 \mathrm{~mm}$ de profundidade. Para o contato elétrico, soldou-se à placa de platina um fio de platina e, este último, foi soldado a um fio de cobre. A representação do eletrodo utilizado encontra-se na Figura 2.

Observação: O grafite deve ser tratado previamente com uma solução de $\mathrm{HCl} 1 \times 10^{-3} \mathrm{~mol} \mathrm{~L}{ }^{-1}$. Agitar essa suspensão e deixar em repouso por aproximadamente $30 \mathrm{~min}$. Posteriormente este material deve ser lavado e filtrado com água bidestilada. Levar o grafite em estufa a $373 \mathrm{~K}$ por aproximadamente 3 h. A quantidade de grafite a ser tratado pelos alunos deve ser suficiente para que todos utilizem este nos experimentos voltamétricos. $O$ tratamento do grafite é importante para que não se observe picos voltamétricos relacionados a impurezas que podem estar presentes neste.

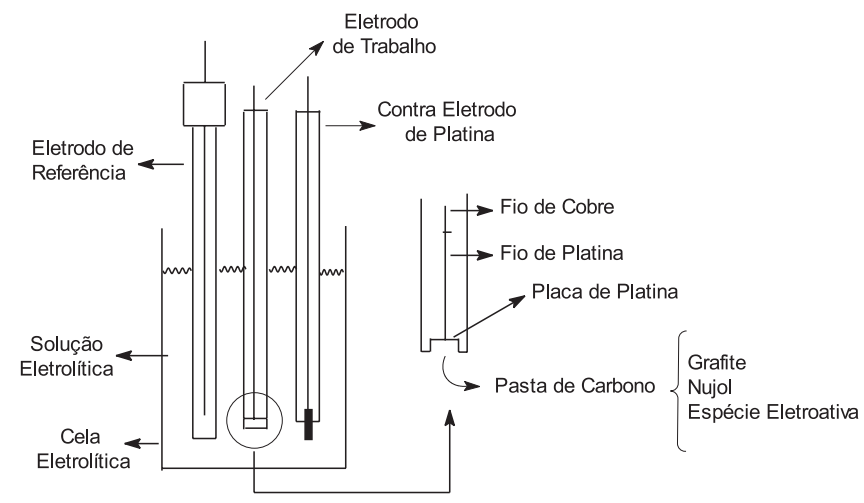

Figura 2. Representação da cela eletroquímica

Registro dos voltamogramas de pulso diferencial

Para o registro dos voltamogramas de pulso diferencial da nbutil(piridil)cobaloxima utilizou-se uma cela eletroquímica (Figura 2) contendo três eletrodos: contra eletrodo de platina, eletrodo de calomelano saturado (ECS) como referência e eletrodo de pasta de carbono modificado com n-butil(piridil)cobaloxima. Os voltamogramas foram registrados em $25 \mathrm{~mL}$ de solução de $\mathrm{KCl} 0,5 \mathrm{~mol} \mathrm{~L}^{-1}$ previamente deaerada com nitrogênio por aproximadamente $30 \mathrm{~min}$. A faixa de potencial empregada foi de $-1,0$ a $1,0 \mathrm{~V}$ e a velocidade de varredura foi de $10 \mathrm{mV} \mathrm{s}^{-1}$. As medidas eletroquímicas foram obtidas em um Potenciostato/Galvanostato PAR 273A, conectado a um microcomputador.

Observação muito importante: A deaeração da cela eletrolítica é importante porque espécies de cobalto possuem uma grande afinidade por oxigênio molecular. Além disso, um pico redox é observado quando o grafite está na presença de oxigênio molecular. Este pico voltamétrico possui um potencial de redução com um valor aproximadamente de $-500 \mathrm{mV}$.

\section{RESULTADOS E DISCUSSÃO}

\section{Síntese da n-butil(piridil)cobaloxima}

No complexo butil(piridil)cobaloxima o íon cobalto encontra-se no estado de oxidação $3+$ e possui 6 elétrons d. Estes elétrons são estabilizados através da coordenação octaédrica com cinco átomos 
de nitrogênio, sendo que quatro destes átomos são referentes a duas moléculas de dimetilglioxima, um da piridina e a sexta coordenação é proveniente da ligação covalente entre este metal e um átomo de carbono.

Para a obtenção do complexo n-butil(piridil)cobaloxima é necessário a obtenção do íon cobalto intermediário no estado de oxidação +1 . Existem dois métodos para a síntese de complexos de cobalto (I). Um deles foi desenvolvido por Schrauser e consiste na utilização de boroidreto de sódio como agente redutor, atuando sob condição de baixa temperatura. Outro método de formação de complexos de cobalto (I) consiste no desproporcionamento alcalino, o qual foi realizado nesta prática e que consiste na adição de uma base forte ao meio reacional, como por exemplo $\mathrm{NaOH}$. É preferível utilizá-lo em relação ao método de Schrauser devido à sua praticidade, rapidez e custo. Esta técnica é representada através da Equação 1. O grupo hidroxila $(O ̈ H)$ do hidróxido de sódio promove o ataque nucleofílico à molécula de dimetilglioxima, resultando no mono ânion dimetilglioximato.

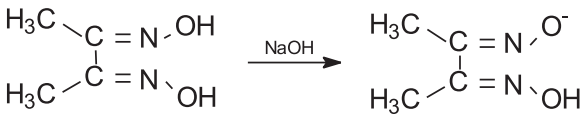

Durante o processo de formação do complexo n-butil(piridil) cobaloxima ocorre a formação da cobaloxima (II) (reação 2) que, em seguida, reage com excesso de base $(\mathrm{NaOH})$. Neste momento ocorre o desproporcionamento resultando na cobaloxima $(\mathrm{I})(\mathrm{dmgH})_{2} \ddot{\mathrm{C}}^{-}$ Py, o qual é considerado um potente nucleófilo, e na cobaloxima (III) $(\mathrm{dmgH})_{2} \mathrm{Co}^{+} \mathrm{Py}$ (reação 3). A cobaloxima (I) reagirá com o 1bromobutano e formar-se-á o complexo n-butil(piridil)cobaloxima (reação 4)

$2 \mathrm{dmgH}_{2}+2 \mathrm{NaOH}+\mathrm{CoCl}_{2}+$ py $\rightarrow(\mathrm{dmgH})_{2} \mathrm{Copy}$

$2(\mathrm{dmgH})_{2}$ Copy $\stackrel{\text { desproporcionamento }}{\longrightarrow}(\mathrm{dmgH})_{2} \mathrm{Co}^{+}$py $+(\mathrm{dmgH})_{2} \ddot{\mathrm{C}} \mathrm{o}^{-\mathrm{Py}}$

$(\mathrm{dmgH})_{2} \ddot{\mathrm{Co}}-\mathrm{Py}+\mathrm{C}_{3} \mathrm{H}_{7}-\mathrm{CH}_{2}-\mathrm{Br} \rightarrow \mathrm{C}_{4} \mathrm{H}_{9}-(\mathrm{dmgH})_{2} \mathrm{Co}^{(\mathrm{III})} \mathrm{py}$

em que py significa o ligante piridina.

É interessante destacar neste caso que a coloração observada durante a síntese do complexo de n-butil(piridil)cobaloxima é devida ao íon cobalto. A variação da cor muda de acordo com a mudança do estado de oxidação do íon cobalto. No caso da coloração azul escuro, o complexo de cobalto encontra-se no estado de oxidação não característico +1 . Portanto, em virtude da sensibilidade do complexo de cobalto (I) ao oxigênio atmosférico, faz-se necessário o uso de atmosfera inerte. A mudança do estado de oxidação do complexo de cobalto (I) intermediário (azul escuro) para o complexo alaranjado escuro indica a formação do complexo de cobalto (III). A variação de cor azul para alaranjado ocorre devido ao ataque nucleofílico do íon cobalto (I) intermediário ao 1-bromobutano. O íon cobalto (I) desloca os íons brometo do 1-bromobutano $\mathrm{R}-\mathrm{Br}(\mathrm{R}=$ alquila) formando a ligação cobalto-carbono e resultando no complexo nbutil(piridil)cobaloxima (III). A mudança dos estados de oxidação para o íon cobalto é sumarizado no Esquema 1.

\section{Caracterização da n-butilcobaloxima}

Espectroscopia na região do infravermelho

As bandas observadas no espectro de infravermelho para a nbutil(piridil)cobaloxima em 2955, 2918 e $2854 \mathrm{~cm}^{-1}$ são atribuídas aos estiramentos dos grupos $-\mathrm{CH}_{3} \mathrm{e}-\mathrm{CH}_{2}$ do ligante butilalcano, como

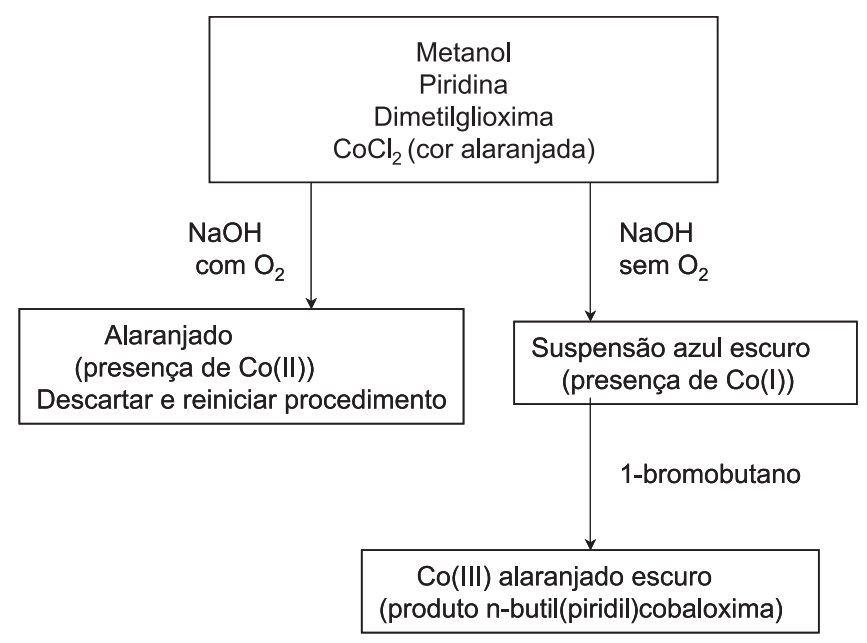

Esquema 1. Representação da mudança dos estados de oxidação do íon cobalto durante a obtenção do complexo n-butil(piridil)cobaloxima

pode ser observado na Figura 3. Isto é uma evidência da formação do complexo n-butil(piridil)cobaloxima, pois indica a ligação do Co(III) com o butilalcano. Além disso, o composto apresenta outras atribuições características como por exemplo $v \mathrm{OH}$ em $3350 \mathrm{~cm}^{-1}$; $\vee \mathrm{C}=\mathrm{N} 1560 \mathrm{~cm}^{-1}$ e $v$ Co-N em $518 \mathrm{~cm}^{-1}$. A Tabela 1 sumariza as atribuições características para a n-butil(piridil)cobaloxima ${ }^{6}$.

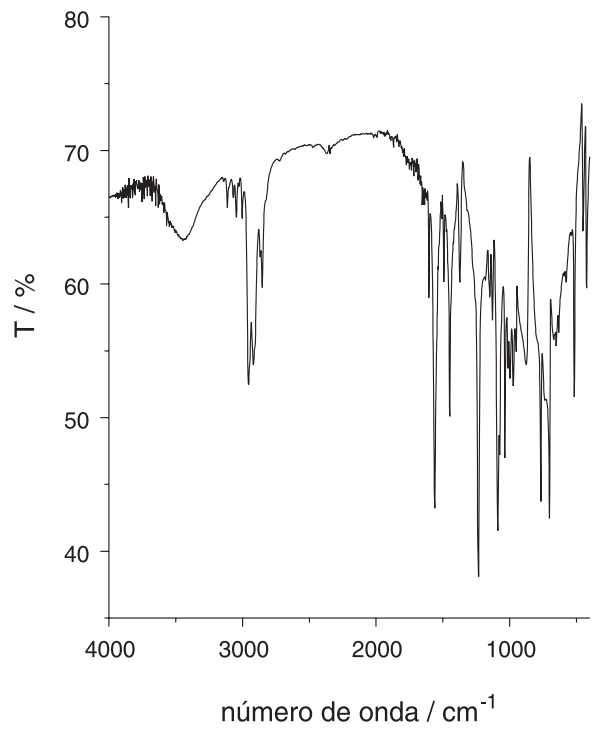

Figura 3. Espectro de infravermelho do complexo n-butil(piridil)cobaloxima

Tabela 1. Freqüências vibracionais $\left(\mathrm{cm}^{-1}\right)$ da n-butil(piridil) cobaloxima

\begin{tabular}{cc}
\hline $\mathrm{n}^{\text {o }}$ de ondas $/ \mathrm{cm}^{-1}$ & Atribuições apropriadas \\
\hline 3350 & $\vee \mathrm{OH}$ \\
$2955,2918,2854$ & $\vee \mathrm{C}-\mathrm{H}$ \\
1600 & $\delta \mathrm{C}-\mathrm{H}, \mathrm{C}=\mathrm{C}$ ou $\mathrm{C}=\mathrm{N}$ do anel piridínico \\
1560 & $\vee \mathrm{C}=\mathrm{N}$ \\
1440,1375 & $\delta \mathrm{CH}$ \\
1240,1090 & $\vee \mathrm{N}-\mathrm{O}$ \\
980,700 & $\delta \mathrm{C}=\mathrm{N}-\mathrm{O}$ e d OH \\
518 & $\vee \mathrm{Co}-\mathrm{N}$ \\
\hline
\end{tabular}




\section{Espectros eletrônicos}

$\mathrm{O}$ espectro na região UV-vis da n-butil(piridil)cobaloxima em solução de acetonitrila encontra-se na Figura 4. O espectro da nbutil(piridil)cobaloxima apresentou uma banda em $240 \mathrm{~nm}$ devido às transições $\pi-\pi^{*}$ do ânion dimetilglioximato e dos ligantes axiais (py e $-\mathrm{C}_{4} \mathrm{H}_{9}$ ). As bandas observadas entre 310 a $380 \mathrm{~nm}$ não foram identificadas de maneira precisa e podem ser provenientes de uma banda de transferência de carga metal-ligante entre o átomo de cobalto e os ligantes dimetilglioximato ou de transições d-d. Cabe ressaltar aqui que as bandas atribuídas à transferência de carga metal-ligante (TCML) ocorrem em complexos em que os ligantes sejam insaturados, contenham orbitais $\pi$ antiligantes (como é o caso do ânion dimetilglioximato) e, além disso, que sejam coordenados a metais de transição oxidáveis (neste caso o íon cobalto). Observouse também no espectro uv-vis uma banda de fraca intensidade em $430 \mathrm{~nm}$ que pode ser atribuída a uma transferência de carga da ligação Co-C. Segundo a literatura, a energia da transição de transferência de carga Co-C surge em virtude do aumento do caráter doador dos ligantes equatoriais e axiais. Com o aumento do caráter doador ocorre também o aumento da densidade de carga sobre o cobalto e, desta forma, estabiliza a ligação Co-C. Entretanto, alguns autores preferem atribuir esta banda às transições $d-d$ de campo ligante ${ }^{7,13-16}$.

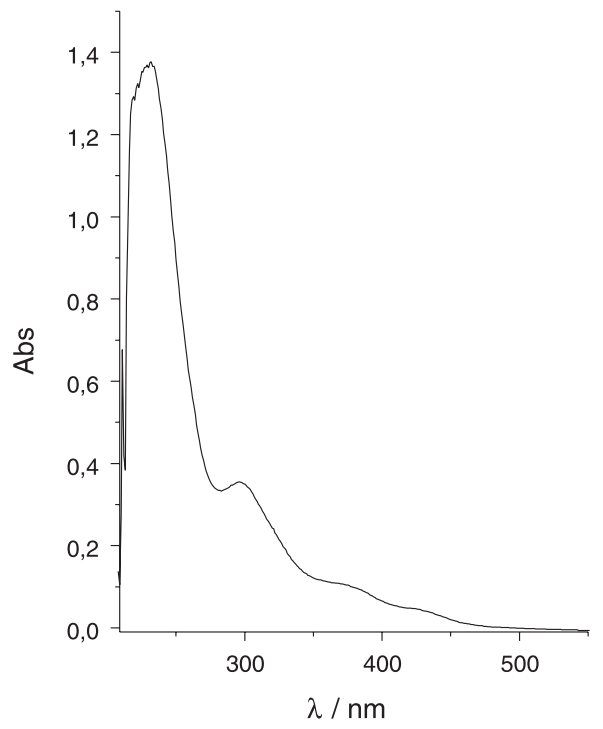

Figura 4. Espectro eletrônico da n-butil(piridil)cobaloxima

Comportamento voltamétrico da n-butil(piridil)cobaloxima (III)

Com o intuito de verificar os estados de oxidação do íon cobalto presente na n-butil(piridil)cobaloxima, registraram-se voltamogramas cíclicos e de pulso diferencial em eletrólito suporte. Não foi observado nenhum processo redox quando se registrou os voltamogramas cíclicos para o complexo. Assim, a voltametria de pulso diferencial foi usada por ser mais sensível que a voltametria cíclica, proporcionando um aumento na sensibilidade da resposta eletroquímica da espécie eletroativa. Em eletrodos sólidos, a espécie eletroativa encontra-se aderida ao eletrodo de trabalho, ao contrário do que se observa nos estudos realizados em solução em que a espécie eletroativa se difunde da solução para a superfície do eletrodo onde ocorre o processo de oxi-redução. Portanto, o eletrodo sólido tornase mais atraente embora a sua confecção o torne trabalhoso ${ }^{17}$.

$\mathrm{O}$ voltamograma de pulso diferencial da n-butil(piridil)cobaloxima é mostrado na Figura 5. Observou-se a presença de dois picos de oxidação, com o primeiro deles sendo registrado em $-423 \mathrm{mV}$ (a) e atribuído ao processo:

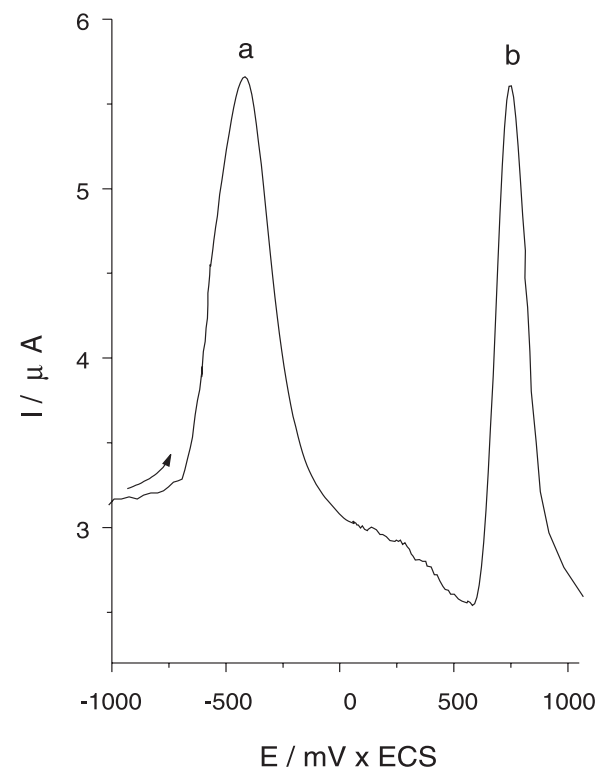

Figura 5. Voltamograma de pulso diferencial da n-butil(piridil)cobaloxima. Processo de oxidação $\mathrm{Co}(\mathrm{I}) \rightarrow \mathrm{Co}(I I)($ a $)$ e $\mathrm{Co}(I I) \rightarrow \mathrm{Co}(I I I)(b)$. Velocidade de varredura: $10 \mathrm{mV} \mathrm{s}^{-1}$. Eletrólito suporte: $\mathrm{KCl}$ 0,5 $\mathrm{mol} \mathrm{L}^{-1}$

$\mathrm{Co}(\mathrm{I}) \rightarrow \mathrm{Co}(\mathrm{II})+\mathrm{e}^{-}$

O segundo pico, registrado em $752 \mathrm{mV}$ (b) corresponde ao processo:

$\mathrm{Co}(\mathrm{II}) \rightarrow \mathrm{Co}(\mathrm{III})+\mathrm{e}^{-}$

O potencial de oxidação do $\mathrm{Co}(\mathrm{II})$ a $\mathrm{Co}(\mathrm{III})$ encontrado em um eletrodo de pasta de carbono modificado com ftalocianina de cobalto foi de $700 \mathrm{mV}^{18}$ e para a ftalocianima de cobalto tetrassulfonada em solução foi de $800 \mathrm{mV}^{19}$. Através desses valores pode-se atribuir que o pico em $752 \mathrm{mV}$ realmente se deve ao processo de oxidação do Co(II) a Co(III). Já o potencial atribuído para a oxidação do Co(I) a Co(II) é muito semelhante ao encontrado na ftalocianina de cobalto tetrassulfonada em solução, que é de $-500 \mathrm{mV}{ }^{20}$ e na cobaloxima em solução de acetonitrila, que é de $-700 \mathrm{mV}^{21}$.

\section{CONCLUSÃO}

Nesta experiência, dirigida ao curso de graduação em química, foram abordados alguns tópicos relacionados aos metais de transição, em especial ao cobalto, em vários estados de oxidação, bem como aspectos importantes como estudos eletroquímicos do metal em questão.

Os estados de oxidação do metal de transição da n-butil(piridil) cobaloxima podem ser visualizados facilmente pela mudança de cor durante o método de preparação. Além disso, a voltametria de pulso diferencial utilizada aqui permite aos alunos a aquisição de conhecimentos em eletroquímica que, geralmente nos cursos de graduação, permanecem restritos à teoria. Constatou-se que é uma experiência relativamente simples e sua execução é de baixo custo.

Coube aos alunos a realização de relatórios e estes foram satisfatórios, pois se comprovou a facilidade na compreensão e no discernimento de vários tópicos abordados, entre eles familiarização com complexos metálicos de cobalto, um precursor modelo para a vitamina $\mathrm{B}_{12}$, ligação metal-carbono, manuseio e conhecimentos so- 
bre os eletrodos de espécies eletroativas no estado sólido e suas vantagens. Além disso, técnicas tradicionais como espectroscopia no infravermelho e no uv-vis também podem ser abordadas.

\section{AGRADECIMENTOS}

C. A. Borgo e A. M. Lazarin agradecem ao CNPq e à FAPESP pelas bolsas de doutoramento recebidas.

\section{REFERÊNCIAS}

1. Brown, T. M.; Cooksey, C. J.; Crich, D.; J. Chem. Educ. 1990, 11, 67.

2. Brown, T. M.; Cooksey, C. J.; Crich, D.; Dronsfield, A. T.; J. Chem. Educ. 1990, 67, 434.

3. Brown, T. M.; Prog. Inorg. Chem. 1973, 18, 235.

4. Schrauzer, G. N.; Inorg. Syn. 1968, 11, 61.

5. Brown, T. M.; Cooksey, C. J.; J. Chem. Educ. 1987, 24, 77.

6. Gannotti, G.; Fontaine, C.; Septe, B.; J. Organomet. Chem. 1974, 71, 107.

7. Schrauzer, G. N.; Lee, L. P.; J. Am. Chem. Soc. 1970, 92, 2997.

8. Ngameni, E.; Ngioume, J.; Nasi, A.; Belombe, M. M.; Roux, R.;
Electrochim. Acta 1996, 41, 2571.

9. Hisaieda, Y.; Nishioka, T.; Inoue, Y.; Asada, K.; Hayashi; T.; Coord. Chem. Rev. 2000, 198, 21.

10. Kissinger, P. T.; Heineman, R. W.; J. Chem. Educ. 1983, 60, 702.

11. Mabbot, G. A.; J. Chem. Educ. 1983, 60, 697.

12. Evans, D. H.; O Connel, K. M.; Petersen, R. A.; Kelly, M. J.; J. Chem. Educ. 1983, 60, 290.

13. Randaccio, L.; Bresciani Pahor, N.; Zangrando, E.; Marzilli, L. G.; Chem. Soc. Rev. 1989, 18, 225.

14. Brown, D. G.; Prog. Inorg. Chem. 1973, 18, 235.

15. Shirotani, I.; Suzuki, K.; Suzuki, T.; Yagi, T.; Tanaka, M.; Bull. Chem. Soc. Jpn. 1992, 65, 1078.

16. Ochiai, E.; Long, K. M.; Sperati, R.; Busch, D. H.; J. Am. Chem. Soc. 1969, 44, 1581.

17. Brett, A. M. O.; Brett, C.; Eletroquímica-Princípios, Métodos e Aplicações, Livraria Almedina: Coimbra, 1996.

18. Huang, X.; Kok, W. T.; Anal. Chim. Acta 1993, 273, 245.

19. Zagal, J. H.; Sem, R. K.; Yeger, E.; J. Electroanal. Chem. 1977, 83, 207.

20. Zecevis, S.; Glavaski, B. S.; Yeager, E.; J. Electroanal. Chem. 1985, 196, 339.

21. Shamsipur, M; Salimi, A.; Haddadzadeh, H.; Mousavi, M. F.; J. Electroanal. Chem. 2001, 517, 37. 